\title{
Linear Modeling of Metrical Poetry Respiratory Signal Yonghong $\mathrm{Li}^{1}$
}

\author{
${ }^{1}$ Key Lab of China's National Linguistic Information Technology, Northwest University for \\ Nationalities, Lanzhou, 730030, China
}

Keywords: Respiratory signal; Poetry; Model

\begin{abstract}
Through analysis of breathing physiological characteristics of Chinese metrical poetry, we studied human speech breathing patterns. We selected four kinds of representative poem: fivecharacter octave, five-character quatrain, seven-character octave, and seven-character quatrain , each types of five, we recorded a speech, voice, respiratory signal and designed a set of parameters of respiratory signal; By using well-established respiratory signal processing platform, we extracted the relevant parameters of poetry respiratory signal and made the linear analog of breathing patterns.
\end{abstract}

\section{Introduction}

Respiration is the driving force and foundation of sound, quiet breathing and breathing when talking is not the same, the expiratory phase and inspiratory phase are similar during quiet breathing, but the expiratory phase is far greater than inspiratory phase during speaking[1](Feng BaoFu,1981). Peking University is the first use of breath bandage to study the characteristics of the respiratory rhythm of Mandarin poems, they designed the respiratory signal processing platform, proposed a set of parameters for the analysis of breathing reset, mainly research the magnitude and duration distribution of breathing reset during reading different styles of modern style poetry of Mandarin, words, novels, essays, news and so on [2][3] (Tan JingJing, Li YongHong, 2008).

Metrical poetry is written by according to a certain format and rules of poetry. It has strict rules of poetry's lines, character number (or syllables), tone and phonology, antithesis, and sentence arrangement, etc. Compared to other type of writing, the rhythm pattern rules are relatively strong and easy to model. In this paper, we choose metric verse as the research object, and selected four kinds of representative poem of five-character octave , five-character quatrain, seven-character octave, and seven-character quatrain , each type of five, the total number is 20, speakers were familiar with the material in order to avoid mistakes of pause and semantic planning during reading. First of all, we collected voice, throat and breathing signals of these 20 poems, and made the breathing marker; Then, by using the improved breathing signal analysis platform, we extracted related parameters of poetry breath cell, and compared breathing pattern of four kind of metrical poetry; Finally, we made a linear model of breathing signal and revealed the internal mechanism of metrical poetry during breathing of reading them.

\section{Respiratory Signal Processing}

\section{Acquisition of respiratory signal}

By using EMG which produced by Australian ADInstruments (ADI) company, tying the breath bandage to speaker's chest, then the sensor of breath bandage can change the length change of breath bandage caused by expanding and contracting of chest into electrical signals, thereby, we can gain the respiratory amplitude signals following the change of time[4].

\section{Parameter setting of respiratory signal}

We all believe that the airflow is eupnea and smooth in poetry reading, atmospheric pressure inside body is consistent with atmospheric pressure of outside, signal is zero, there would be a process of deep breathing in reading the first sentence of poetry, signals from zero up to the peak point, with the reduction of body airflow the pressure reduction and signal slow down in the process of reading a poem, after reading, signals down to the valley point, and we finished the first breath 
cell, then repeat the breathing process of preparing for the second sentence of poetry reading, until the whole poem recital finished, while the breathing process end.

A full breathing process is defined as Breath Cell, in contains inspiration cell and expiration cell. Respiratory signal curve rises as the inspiration process, generally it corresponds to the silent segment of speech signals, the signal decreases as expiration process that generally corresponds to the voice segment of speech signals. The main computation breathe cell parameter in Fig.1 and Table 1.

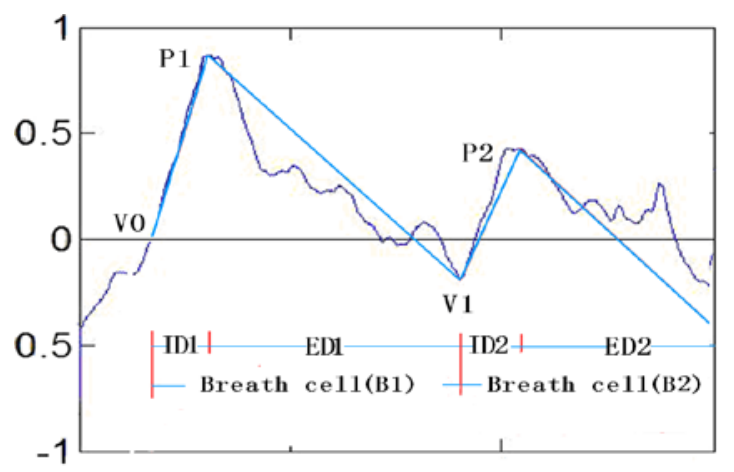

Fig.1: P-Diagram of poetry respiratory signal

Table 1: Parameter of respiratory signal

\begin{tabular}{lll}
\hline Inspiration Cell(I) & Expiration Cell(E) & Breath Cell(B) \\
\hline duration : ID & duration: ED & duration:BD BD(n)=ID(n)+ED(n) \\
peak value: & valley value: $\mathrm{V}$ & total amount of airflow: BQ; \\
slope:IK: & slope: EK: & BQ(n)=IQ(n)+EQ(n) \\
IK(n)= IQ(n)/ ID & EK(n)=-EQ(n)/ED & \\
inspiratory capacity: IQ; & expiratory capacity: EQ ; & \\
IQ(n)= P(n)-V(n-1) & EQ(n)= P(n)-V(n) & \\
\hline
\end{tabular}

The peak point $\mathrm{P}(\mathrm{n})$ is a transition point of the inspiratory and expiratory in respiratory signal; $\mathrm{V} 0$ as the starting point of inspiration the value is zero; Valley point $\mathrm{V}(\mathrm{n})$ is a transition point of the inspiratory and expiratory, also is dividing point of breath cell. Duration is the time which used in inspiration cell and expiration cell. Inspiratory capacity is the magnitude difference of inspiration cell from peak point to valley point, which means the volume of the airflow human inhaled, expiratory capacity is the magnitude difference of expiration cell from peak point to valley point, which means the volume of the airflow human exhaled during reading, and the total volume of airflow represents the airflow of breath cell. Slope means the changing speed of inspiratory capacity and expiratory capacity, the greater the absolute value of the slope, the faster speed of airflow.

\section{Respiratory Signal processing}

The designed the speech signal analysis platform achieves variety of functions such as automatically or manually tagging, parameter extraction, and systematic analysis of speech signal, which provides a good technical support for deeper research of speech production and speech rhythms. Main functions are: 1) the use of low-pass smoothing filter high-frequency noise and some slight interference signal; 2) the use of local maximum method, automatic testing the location of the signal peaks, supplemented by manual modification and adjustment; 3) extract respiratory signal parameters by tag information, and save it to the xls file. Figure 2 is the sample map of poetry breathing markers, five-character quatrain (up), five-character octave (upper middle), sevencharacter quatrain (lower middle), seven-character octave (below). 


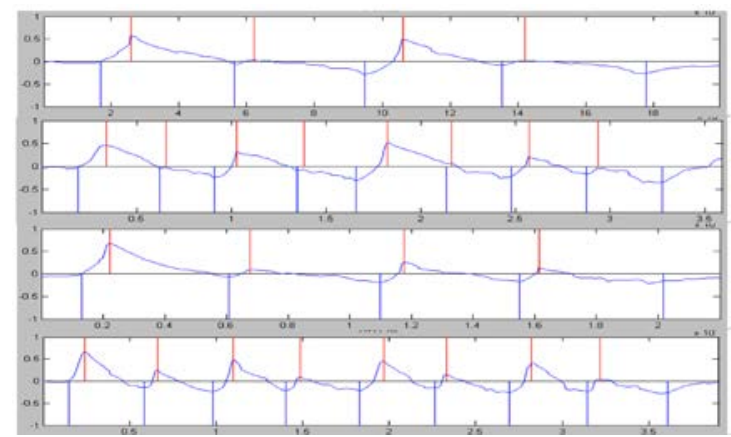

Fig.2: Poetry breathing markers

\section{Poetry breathing signal analysis}

In the reading process, speakers can conscious control of breathing pattern according to the need of expression. On the whole, poetry breathing signal is divided into two categories, jue ju (a poem of four lines, each containing five or seven characters, with strict tonal pattern and rhyme scheme), and lv shi (a classical poem of eight lines, each containing five or seven characters, with strict tonal pattern and rhyme scheme ).

\section{Five-character quatrain}

A poem of four lines, each containing five characters and total has twenty characters of each poem. We selected five poems as sound corpus, they were "Jiang Xue," "Deng Guanquelou", "Min Nong," "Chun Xiao" and "Jing Yesi", as recording corpus, we extracted breath cell parameters of each poem and conducted an average value of parameters (see Table 2).

Table 2: Breath parameters of Five-Character Quatrain

\begin{tabular}{ccccccccccc}
\hline No. & \multicolumn{4}{c}{ Inspiration Cell(I) } & \multicolumn{4}{c}{ Expiration Cell(E) } & \multicolumn{3}{c}{ Breath Cell (B) } \\
\cline { 2 - 11 } & ID & P & IQ & IK & ED & V & EQ & EK & BD & BQ \\
\hline 1 & 1.19 & 0.61 & 0.61 & 0.51 & 3.06 & -0.03 & 0.64 & 0.21 & 4.26 & 1.24 \\
2 & 0.59 & 0.04 & 0.07 & 0.12 & 3.04 & -0.23 & 0.27 & 0.09 & 3.63 & 0.34 \\
3 & 1.10 & 0.47 & 0.71 & 0.64 & 2.87 & -0.12 & 0.59 & 0.21 & 3.97 & 1.29 \\
4 & 0.63 & -0.06 & 0.05 & 0.08 & 3.74 & -0.27 & 0.20 & 0.05 & 4.38 & 0.26 \\
\hline
\end{tabular}

The breathing rhythm of five-character quatrains is very regular. Respiratory signal of whole poem is divided into four distinct ups and downs. The first sentence and the third sentence as the beginning of each sentence, inspiratory section value $P$, inspiratory capacity (IQ) and inspiratory duration (ID) are larger than the second sentence and the fourth sentence. Because the words in each sentence are same, the duration of inspiratory section are not vary much, however, the fourth sentence as the end of a poem, which has the phenomenon of drag and the longest exhalation duration, the minimum value $\mathrm{V}$ and the largest negative pressure of human body.

\section{Seven-character quatrain}

A poem of four lines, each containing seven characters and total has twenty eight characters of each poem, as five-character quatrain both belongs to modern style poetry. We Selected five poems of Du Fu's "Jue Ju" as recorded corpus, we extracted breath cell parameters of each poem and conducted an average value of parameters (see Table3).

Table 3: Breath Parameters of Seven-Character Quatrain

\begin{tabular}{ccccccccccc}
\hline No. & \multicolumn{4}{c}{ Inspiration Cell(I) } & \multicolumn{4}{c}{ Expiration Cell(E) } & \multicolumn{3}{c}{ Breath Cell (B) } \\
\cline { 2 - 11 } & ID & P & IQ & IK & ED & V & EQ & EK & BD & BQ \\
\hline 1 & 0.95 & 0.59 & 0.59 & 0.62 & 3.83 & -0.08 & 0.68 & 0.18 & 4.78 & 1.27 \\
2 & 0.60 & 0.10 & 0.18 & 0.31 & 3.88 & -0.18 & 0.28 & 0.07 & 4.48 & 0.47 \\
3 & 0.95 & 0.34 & 0.52 & 0.54 & 3.56 & -0.16 & 0.50 & 0.14 & 4.51 & 1.01 \\
4 & 0.54 & 0.05 & 0.21 & 0.38 & 4.09 & -0.24 & 0.28 & 0.07 & 4.63 & 0.49 \\
\hline
\end{tabular}


Although the number of words in each sentence of seven-character quatrain is slightly longer than the five-character quatrain, from the whole, breathing pattern of these two kinds of poetry are same. In the second and fourth sentence, the inspiration cell's inspiratory capacity in seven Characters quatrain is greater than seven Characters quatrain's, and breathing pattern is more independent.

\section{Five-character octave}

Five-character octave is a kind of modern style poetry, which has a very strict order in rules and forms, there are stringent regulations in poetry's lines, character number (or syllables), tone and phonology, antithesis, and sentence arrangement, etc. A poem usually has eight lines, every two into a unite, totally it has four unites. Each sentence contains five characters, totally has forty characters in each poem. We selected five poems of "Wang Yue", "Yue Ye Shuhuan", "Chun Wang," "Tian Mo Huai Li Bai" and "Yue Ye" to analysis breathing signals (see Table 4).

Table 4: Breath Parameters of Five-Character Octave

\begin{tabular}{ccccccccccc}
\hline No. & \multicolumn{4}{c}{ Inspiration Cell(I) } & \multicolumn{4}{c}{ Expiration Cell(E) } & \multicolumn{2}{c}{ Breath Cell (B) } \\
\cline { 2 - 11 } & ID & P & IQ & IK & ED & V & EQ & EK & BD & BQ \\
\hline 1 & 1.11 & 0.50 & 0.50 & 0.45 & 5.86 & -0.20 & 0.70 & 0.12 & 6.98 & 1.19 \\
2 & 1.09 & 0.26 & 0.46 & 0.42 & 5.75 & -0.28 & 0.74 & 0.13 & 6.83 & 1.19 \\
3 & 1.36 & 0.49 & 0.76 & 0.56 & 5.84 & -0.16 & 0.93 & 0.16 & 7.20 & 1.69 \\
4 & 0.96 & 0.22 & 0.39 & 0.40 & 6.35 & -0.26 & 0.48 & 0.08 & 7.31 & 0.87 \\
\hline
\end{tabular}

Due to the five-character octave have more sentences, and each sentence have less characters, so the breathing pattern is relatively complex, besides the last unit is not stable, the first three units only have one breath cell, breathing pattern between five-character quatrain and seven-character octave. In order to facilitate analysis the breath signals, we processed breath signals by in accordance with each breath cell of each unit.

\section{Seven-character octave}

Each poem have eight sentences, each sentences contains sever characters, the total characters of each poem is fifty six. We analyzed five poems’ breath signals of "Ke Zhi", "Deng Gao", "Deng Lou," "Shu Xiang" and "Wen Guan Jun Shou Henan Hebei" (see Table 5).

Table 5: Breath Parameters of Seven-Character Octave

\begin{tabular}{ccccccccccc}
\hline No. & \multicolumn{4}{c}{ Inspiration Cell(I) } & \multicolumn{4}{c}{ Expiration Cell(E) } & \multicolumn{3}{c}{ Breath Cell (B) } \\
\cline { 2 - 11 } & ID & P & IQ & IK & ED & V & EQ & EK & BD & BQ \\
\hline 1 & 1.03 & 0.59 & 0.59 & 0.57 & 3.37 & -0.19 & 0.77 & 0.23 & 4.40 & 1.36 \\
2 & 1.18 & 0.14 & 0.33 & 0.28 & 3.52 & -0.22 & 0.55 & 0.15 & 4.70 & 0.87 \\
3 & 1.81 & 0.39 & 0.61 & 0.33 & 3.71 & -0.19 & 0.80 & 0.21 & 5.52 & 1.40 \\
4 & 1.75 & 0.09 & 0.28 & 0.16 & 4.23 & -0.22 & 0.50 & 0.12 & 5.98 & 0.78 \\
1 & 2.58 & 0.46 & 0.67 & 0.26 & 4.43 & -0.18 & 0.86 & 0.19 & 7.01 & 1.53 \\
2 & 2.38 & 0.13 & 0.31 & 0.13 & 4.95 & -0.24 & 0.55 & 0.11 & 7.33 & 0.86 \\
3 & 3.13 & 0.53 & 0.76 & 0.24 & 5.02 & -0.17 & 0.93 & 0.19 & 8.15 & 1.70 \\
4 & 3.09 & 0.12 & 0.29 & 0.09 & 6.03 & -0.24 & 0.53 & 0.09 & 9.12 & 0.82 \\
\hline
\end{tabular}

The difference between seven-character octave and five-character octave is the former have a full breath cell in each sentence. In each unit, the inspiration unit of first sentence is much larger than the second sentence. In each sentence, the inspiration units and expiration units are basic symmetric. The duration of the inspiration units and expiration units are shown as linear growth mode with the increase of sentence number. 


\section{Summary}

From a macro perspective, the inner constraints of rhythm formed a unique breathing pattern of poetry; the main features of breath are as follows:

i) Expect the Wuyan Poems, all the other poems have a large duration of inspiration cell, peak values and inspiratory capacity of first sentence in each unit, we call them as first class expiration cell, and the second sentence of each unit as the second class expiration cell (see Figure 4).

ii) The duration of inspiration cell are related with the word number of poem, which are more stable. Generally, the last sentence as ending also has a phenomenon of dragging and the longest duration of expiration.

iii) The absolute slope values of the inspiration cell and the expiration cell in different style of poems have very good consistency between.

iv) Inspiration rate is generally higher than expiration rate, that is: IK (n)> $|E K(n)|$.

$\mathrm{v}$ ) In general, the peaks of expiration cell $\mathrm{P}>0$, the body pressure is positive, if the valley point $\mathrm{V}<0$, the body pressure is negative.

\section{The mean linear model of respiratory signal}

According to the previous section, we draw the breath signal linear model diagram according to the mean values of breath cell's duration and amplitude, as shown in Figure 8, the horizontal units (seconds).
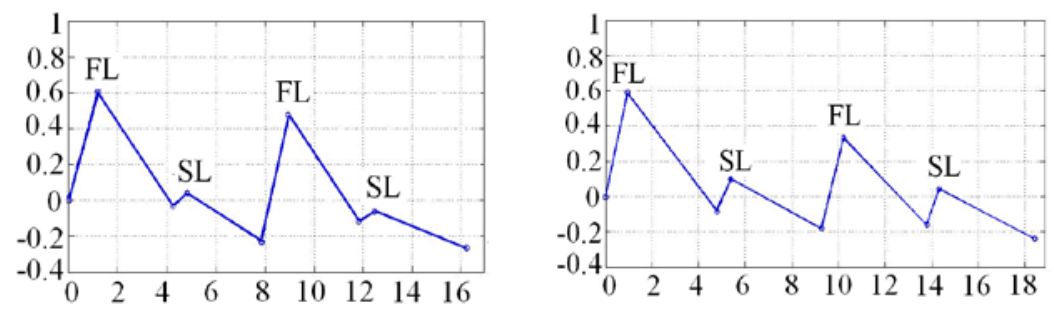

a five-character quatrain

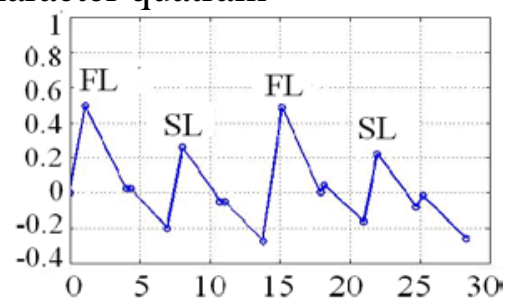

b seven-character quatrain

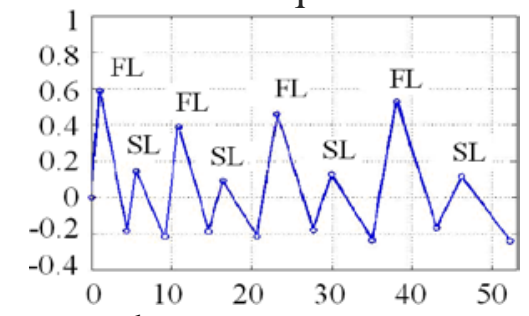

c five-character octave

d seven-character octave

Fig3: Linear model diagram of Metrical Poetry respiratory signal

In Figure 3, respiratory signal models as several units, in each unit, the inspiration units of first sentence is much larger than the second sentence, and the absolute value of slope in first sentence is much bigger than the second sentence. In addition, the three former units all have one breath cell except the last unit in five-character octave, breathing pattern similar to the five-character quatrain. The inspiration units and expiration units of seven-character octave are basic symmetric, 8 breath cell are all presented as "an isosceles triangle mode". Therefore, poetry breathing pattern can be linear simulated as two-stage breath cell.

$$
\mathrm{Y}=\mathrm{kx}+\mathrm{b}
$$

$\mathrm{K}$ means inspiration of inspiration units and slope of expiration units (IK, EK); b means intercept of inspiratory units and expiratory units intercept (IB, EB). Two-stage breath cell total have 8 parameters, the results shown in Table $\mathrm{V}$.

Five-character quatrains, seven-character quatrains and seven-character octave are each sentence with one breath cell, each unit forms one basic breath model, five-character octave for each unit one breath cell, and each two units forms one basic breath model, the four basic models of poetry respiratory signals as shown in Table6. 
Table 6: Coefficient of Poetry Breathing Model

\begin{tabular}{ccccc}
\hline Coefficient & $\begin{array}{c}\text { Five-character } \\
\text { quatrains }\end{array}$ & $\begin{array}{c}\text { Seven-character } \\
\text { quatrains }\end{array}$ & $\begin{array}{c}\text { Five-character } \\
\text { octave }\end{array}$ & $\begin{array}{c}\text { Seven-character } \\
\text { octave }\end{array}$ \\
\hline IK1 & 0.51 & 0.62 & 0.45 & 0.57 \\
IB1 & 0 & 0 & 0 & 0 \\
EK1 & -0.21 & -0.18 & -0.04 & -0.23 \\
EB1 & 0.86 & 0.76 & 0.54 & 0.82 \\
IK2 & 0.12 & 0.31 & 0.21 & 0.28 \\
IB2 & -0.53 & -1.55 & -1.19 & -1.40 \\
EK2 & -0.09 & -0.07 & -0.05 & -0.10 \\
EB2 & 0.47 & 0.49 & 0.85 & 0.71 \\
\hline
\end{tabular}

\section{Conclusions}

In this paper, we analysed the respiratory rhythm pattern of twenty poems, and got the linear mean model, but the linear model is a rough one which we cannot reproduce the real internal characteristics of breathing signal. If we simulate nonlinear modelling of breathing signal, it is not easy to realize, because the internal characteristics of respiratory signal not only related the articles or poetry itself, but also influenced by personal habits, it has a certain degree of arbitrariness, so it is difficult to establish physiological model of respiratory by a statistical algorithms. In addition, chest breath signal is just a local representation for breathing mechanism during reading, we need to combine movement of abdominal muscles in order to better explain and simulated dynamic mechanism of human language.

\section{Acknowledgements}

This article is subsidized by The National Natural Science Foundation of China (Grant No. 61202187).

\section{References}

[1] Feng Bao-Fu, Ji Zhong-Zheng, Liu Yun-Chi, Singing medical basis J, Shanghai Science and Technology Press,1981.

[2] Tan Jing-jing, Li Yong-hong, Kong Jiang-ping ,. A study on breathing-reset in different types of literature-reciting J. Journal of Tsinghua University, 48(S1), pp.910-918, April 2008.

[3] TanJingJing, The study of respiratory rhythm during news reading The journal of Chinese phonetics, Beijing: Commercial Press, 1(1), pp.156 - 163, April 2008

[4] Li Yong-Hong, Yu Hong-Zhi, Kong Jiang-Ping, The research of modern phonetic apparatus and physiological phonetics Life Science Instruments, 6, pp.54 - 58,2008. 